\title{
Wideband Dual Circularly Polarized Antenna for Intelligent Transport Systems
}

\author{
Lehu Wen, Steven Gao, Fellow, IEEE, Qi Luo, Senior Member, IEEE, \\ Wei Hu, Member, IEEE, and Yingzeng Yin, Member, IEEE
}

\begin{abstract}
A wideband dual circularly polarized (DCP) antenna is presented for intelligent transport system (ITS) applications, which can be used to improve the receiver sensitivity and communication quality of ITS. The presented DCP antenna is composed of an orthogonal power divider (OPD) with two orthogonal input ports, four phase shifters for quadrature phase output, and four crossed dipoles for DCP radiation. Detailed equivalent circuit analysis shows that the OPD has two orthogonal inputs and four equal magnitude in-phase and out-of-phase outputs. To achieve two sets of orthogonal quadrature output signals for DCP radiation, the lumped element based differential right-hand transmission line unit cell and left-hand transmission line unit cell are elaborately introduced as the $\pm 45^{\circ}$ phase shifters, and incorporated into the OPD. Eventually, orthogonal quadrature signals are successfully obtained and fed to the crossed dipoles for DCP radiation. The proposed antenna was then designed, fabricated, and measured for ITS applications. The measured results show that the overlapped impedance bandwidth of both two input ports is 1.07-1.85 GHz (53.4\%), and the isolation is higher than $15.2 \mathrm{~dB}$. Moreover, low axial ratio $(<1.7 \mathrm{~dB})$ and symmetrical radiation patterns are achieved for unidirectional DCP radiation.
\end{abstract}

Index Terms-Dual circular polarization, intelligent transport system, wideband antenna.

\section{INTRODUCTION}

$\mathrm{W}$ ITH the rapid development of modern wireless communication systems, vehicle communication techniques have been drawn more and more attentions. Introducing the vehicle wireless communication techniques into the intelligent transport system (ITS), many new functions such as traffic advisories, road emergency alerts, and safety warnings can be easily realized. The real-time information can be further shared by the drivers and other users to improve the traffic efficiency and avoid possible dangers [1]-[3].

As a key component in the vehicle communication systems, the antenna plays an important role at the front-ends of the communication systems. Fig. 1 shows the typical application scenario of the vehicle communication antenna in the ITS. The

This work was supported in part by China Research Institute of Radiowave Propagation, in part by EPSRC grants EP/N032497/1, EP/P015840/1, and EP/S005625/1, and in part by China Scholarship Council. (Corresponding author: Le-Hu Wen.)

Copyright (c) 2015 IEEE. Personal use of this material is permitted. However, permission to use this material for any other purposes must be obtained from the IEEE by sending a request to pubs-permissions@ @ieee.org.

L.-H. Wen, S. Gao, and Q. Luo are with the School of Engineering and Digital Arts, University of Kent, Canterbury, CT2 7NT, U.K. (e-mail: 1w347@kent.ac.uk)

W. Hu and Y. Yin are with the National Key Laboratory of Antennas and Microwave Technology, Xidian University, Xian, 710071, China.

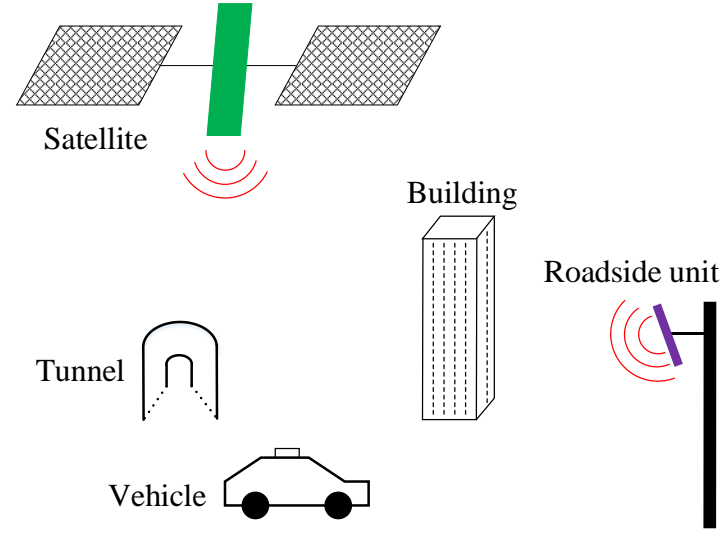

Fig. 1. Application scenario of the vehicle communication antenna in the ITS

antenna in the vehicle receives and obtains the navigation signals from the satellites, and then sends its position information and exchanges related traffic information to the roadside unit that is located on the road as part of the ITS infrastructure [4]-[6]. Because of the advantage of the natural immunity to the orientation angle between the receiving and transmitting antennas, most of these navigation signals are right-hand circularly polarized [7]-[8]. However, due to the multi-path and Faraday rotation effects, such as the multi-reflections from the skyscrapers and shadings by the highway cross bridges and tunnels especially in the downtown area, the received navigation signals become very weak, and even seriously distorted and hard to identify. In addition, the communications between the vehicles and RSUs can also be seriously affected by these various surroundings [9]-[14]. To overcome these problems and ensure high communication quality of the ITS, dual circularly polarized (DCP) antennas, with both right-hand circularly polarized (RHCP) and left-hand circularly polarized (LHCP) radiations, are necessarily required to be integrated into the vehicles and RSUs. Owing to the advantages of the polarization diversity and frequency reuse, the channel capacity and receiver sensitivity in the ITS can be greatly enhanced by using DCP antennas [15].

Because of the advantages of those mentioned above, various techniques are reported to realize DCP antennas recently [16]-[23]. By using the vertically coupled resonator-based structure and introducing the neutralization line between two coupling paths, a low profile high isolated DCP patch antenna [16] is realized. Another convenient way to realize DCP antenna is using the series-fed method to excite the patch with one input port for RHCP radiation, and another for LHCP 
radiation [17]-[18]. However, it is difficult to develop wideband DCP antennas by using patches, especially for the series-fed patch antennas. In addition, with the help of the wideband quadrature couplers, wideband DCP antennas are developed [19]-[20]. However, wideband couplers will introduce additional insertion loss and fabrication cost. The most common wideband low-cost DCP antennas are the monopole antennas and slot antennas [21]-[23]. By using the stepped impedance feed line and U-shaped slot, overlapped impedance and AR bandwidth of $110.5 \%$ is achieved in [23]. However, these antennas normally have bidirectional radiations with one direction for RHCP radiation and the other for LHCP radiation. In addition, unstable radiation patterns are observed for these antennas. The maximum radiation direction varies with the working frequency, and the radiation patterns are asymmetrical with regard to the maximum radiation direction. These unstable radiation characteristics are undesirable for ITS applications.

In this paper, a wideband DCP antenna is presented with unidirectional and symmetrical radiation patterns for ITS applications, which can be used at the front-end of the receiver in the vehicle or the front-end of the RSU to improve the system sensitivity and communication capacity. In the antenna design, an integrated feed network is elaborately designed to provide two sets of equal magnitude and quadrature phase to the crossed dipoles for DCP radiation. The integrated feed network is composed of an orthogonal power divider (OPD), two $+45^{\circ}$ phase shifters, and two $-45^{\circ}$ phase shifters. To realize two sets of wideband quadrature phase excitations for DCP radiation, lumped element based differential left-hand transmission line (LH-TL) unit cell and right-hand transmission line (RH-TL) unit cell are elaborately incorporated into the OPD with $\pm 45^{\circ}$ phase shift. Benefiting from the LH-TL and RH-TL unit cells, wide quadrature bandwidth and small phase variance within $6^{\circ}$ are obtained from $1 \mathrm{GHz}$ to $2 \mathrm{GHz}$. Detailed work principle of the integrated feed network for DCP radiation is illustrated by its equivalent circuit analysis. To validate the design method, the presented DCP antenna is designed, fabricated, and measured. Both the measured and simulated results show that the presented antenna has a wide impedance and AR bandwidth of $53.4 \%(1.07-1.85 \mathrm{GHz})$ and high port isolation $(>15.2 \mathrm{~dB})$. Moreover, low AR $(<1.7 \mathrm{~dB})$ and unidirectional symmetrical radiation patterns are achieved for DCP radiation.

\section{Antenna Configuration}

The detailed configuration of the proposed DCP antenna is shown in Fig. 2, which is composed of the antenna radiator printed on the top and bottom layers of the substrate, two coaxial cables used to feed the antenna, and a square copper sheet as the antenna reflector. In Fig. 2 (a), port 1 is excited for RHCP radiation, while port 2 is excited for LHCP radiation. A commercially available substrate of Rogers $4003 \mathrm{C}$ with the relative dielectric permittivity of 3.55 and the thickness of $0.813 \mathrm{~mm}$ is used for the antenna design.

Fig. 2 (b) shows the detailed configuration of the antenna radiator. Four crossed dipoles with the same arm length $\mathrm{L} 1$ and width $\mathrm{W} 1$ are printed at the peripheral of the bottom layer, and

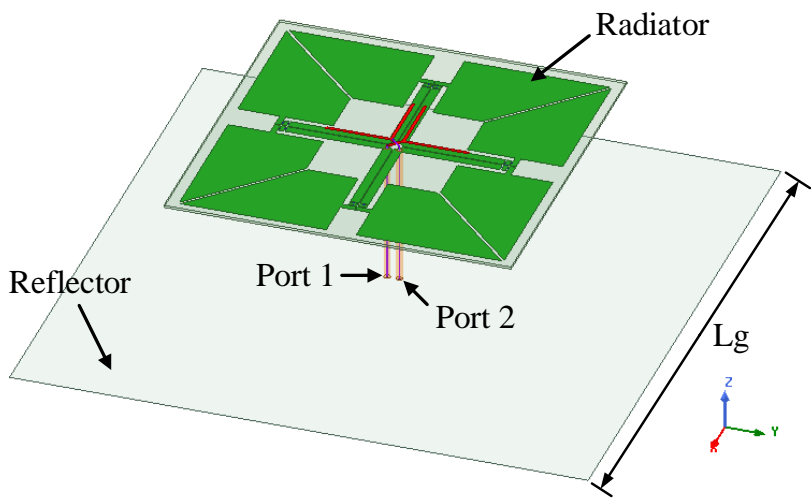

(a)

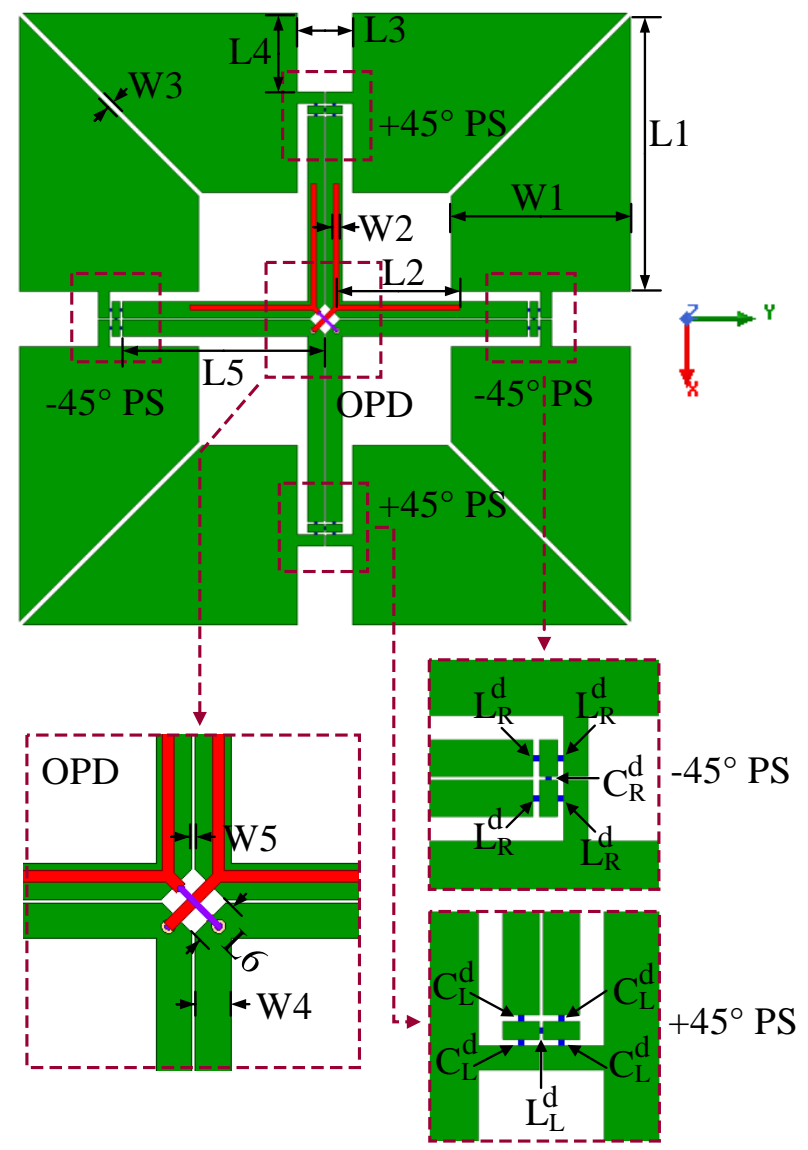

(b)

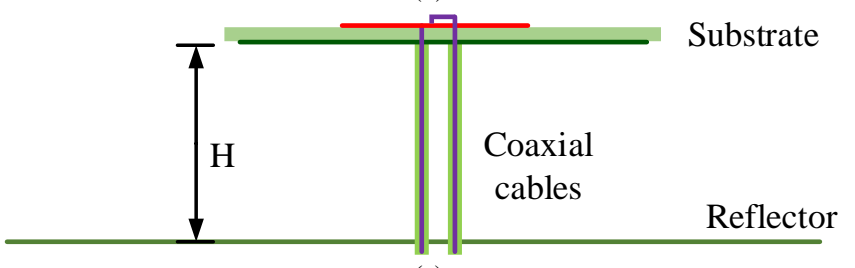

(c)

Fig. 2. Detailed configuration of the proposed DCP antenna. (a) 3D view. (b) Detailed view of the antenna radiator. (c) Side view of the antenna. (Detailed parameters of the antenna, $\mathrm{Lg}=200 \mathrm{~mm}, \mathrm{~L} 1=50.3 \mathrm{~mm}, \mathrm{~L} 2=22.6 \mathrm{~mm}, \mathrm{~L} 3=10.3$ $\mathrm{mm}, \mathrm{L} 4=14.5 \mathrm{~mm}, \mathrm{~L} 5=37.2 \mathrm{~mm}, \mathrm{~L} 6=4 \mathrm{~mm}, \mathrm{~W} 1=33 \mathrm{~mm}, \mathrm{~W} 2=1 \mathrm{~mm}, \mathrm{~W} 3=1$ $\mathrm{mm}, \mathrm{W} 4=3 \mathrm{~mm}, \mathrm{~W} 5=0.3 \mathrm{~mm}, \mathrm{H}=50 \mathrm{~mm}, L_{R}^{d}=2.5 \mathrm{nH}, C_{R}^{d}=0.2 \mathrm{pF}, C_{L}^{d}=2.5 \mathrm{pF}$, $L_{L}^{d}=15 \mathrm{nH}$. PS: Phase shifter.)

are shown in green color. By adjusting L1 and W1, the resonances of the crossed dipoles are changed accordingly. L3 is the feed distance between the two dipole arms, which 


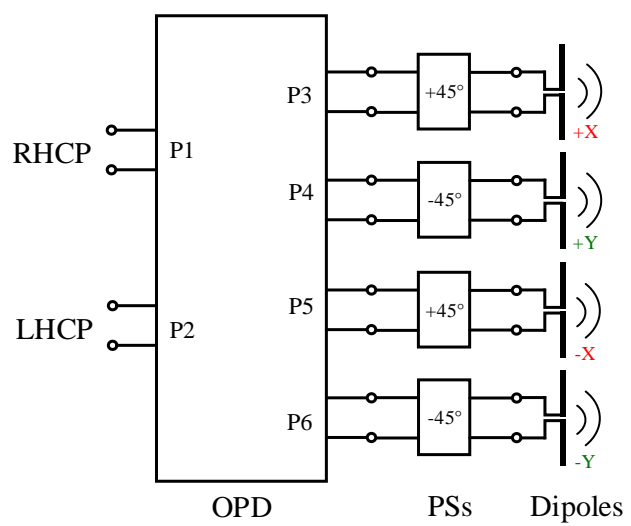

Fig. 3. Equivalent circuit of the proposed DCP antenna. (PSs: phase shifters)

provides enough space for the center integrated feed network. L4 is the feed position on the dipole arm, which is normally set as half of the dipole width. The integrated feed network is printed at the center of the bottom layer, which is composed of an OPD and four lumped element based phase shifters. The OPD is one of the key components of the integrated feed network for DCP radiation, which is composed of four pairs of coupled coplanar striplines on the bottom layer, and two crossed Y-shaped strips on the top layer for excitation. The coupled coplanar striplines have the width W4, edge distance W5, and length L5. Parameters W4 and W5 determine the characteristic impedance of the coplanar stripline. The crossed Y-shaped strips with the width W2 and length L2 work as the open-circuited stubs, which can provide enough capacitance to couple the balanced energy for four coplanar striplines. The phase shifters are the another key component of the integrated feed network, and realized by the T-type differential RH-TL unit cell and T-type differential LH-TL unit cell for $-45^{\circ}$ phase shift and $+45^{\circ}$ phase shift, respectively. The differential RH-TL unit cell consists of four inductors $L_{R}^{d}$ and a capacitor $C_{R}^{d}$, and the differential LH-TL unit cell consists of four capacitors $C_{L}^{d}$ and an inductor $L_{L}^{d}$. Two different types of RH-TL unit cells and LH-TL unit cells are used in this design to keep the radiator symmetrical and compact. The radiator structure on the bottom layer of the substrate is symmetrical with regard to both the $\mathrm{x}$-axis and $\mathrm{y}$-axis. By exciting different coaxial cables, DCP radiation is realized with the incorporation of the elaborately designed OPD and $\pm 45^{\circ}$ phase shifters.

Fig. 2 (c) shows the side view of the proposed DCP antenna. The height between the radiator and the reflector is chosen to be $50 \mathrm{~mm}$ for wideband and unidirectional radiation. Note that the inner conductor of the coaxial cable for RHCP radiation is directly soldered to the top feed line, while the inner conductor of the coaxial cable for LHCP radiation is bent across the RHCP feed line to avoid electrical intersection. Then, it is soldered to the crossed feed line for LHCP radiation. The simulation in this work is obtained from the 3D electromagnetic simulation software ANSYS HFSS. All the detailed parameters of the antenna are listed in the caption of Fig. 2.

\section{WORKING PRINCIPLE}

To illustrate the working principle of the proposed DCP antenna, the equivalent circuit of the antenna is shown in Fig. 3.
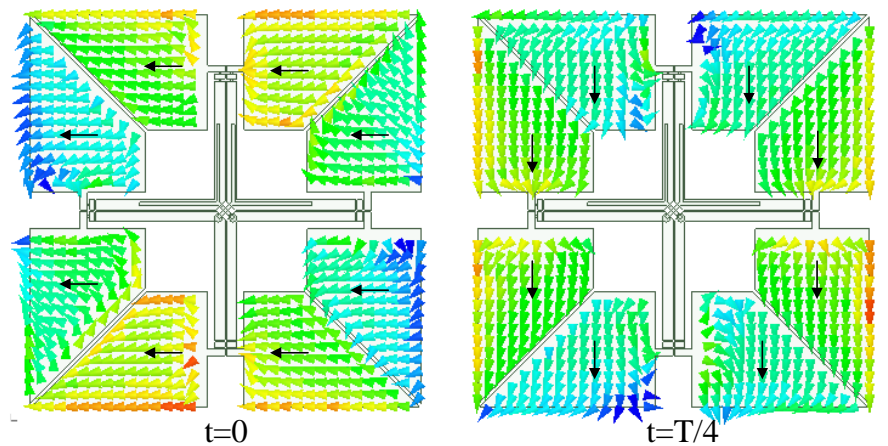

(a)
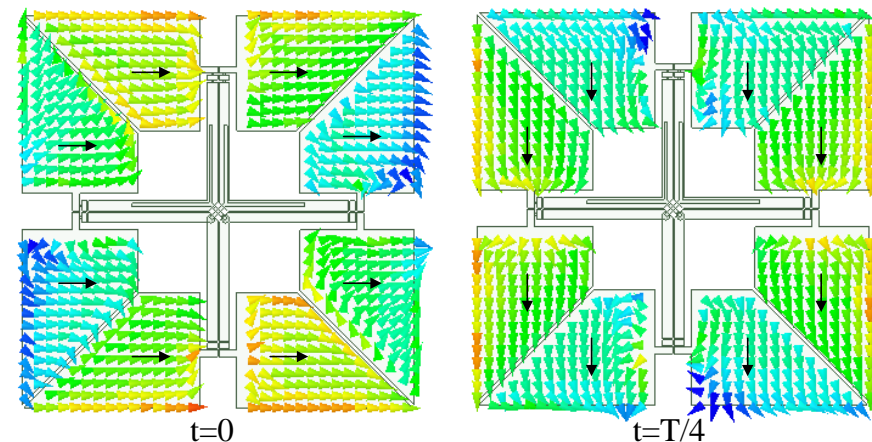

(b)

Fig. 4. Surface current distributions of the proposed antenna at different times of the oscillation period when (a) port 1 is excited and (b) port 2 is excited.

The proposed antenna is composed of the integrated feed network (including an OPD and four phase shifters) and four crossed dipoles. The OPD has two orthogonal input ports with high isolation and four output ports with equal magnitude in-phase and out-of-phase output. After cascading two $+45^{\circ}$ and two $-45^{\circ}$ phase shifters, two sets of orthogonal outputs with equal magnitude and quadrature phase are achieved. Therefore, DCP radiation can be achieved by exciting the crossed dipoles located on the $\pm x$ and $\pm y$ axis, respectively.

Fig. 4 shows the surface current distributions on the crossed dipoles at different times of the oscillation period when input port 1 and port 2 are respectively excited. In the figure, $T$ is the oscillation period at the frequency of $1.4 \mathrm{GHz}$. Auxiliary arrows are added to show the direction of the current. In Fig. 4 (a), at the time $\mathrm{t}=0$, the top and bottom dipoles are mainly excited with strong current magnitude, whereas the left and right dipoles are shown with relatively weak current magnitude. The collective current direction is to the left. At the time $t=T / 4$, the left and right dipoles are excited with strong current magnitude, and the top and bottom dipoles are shown with relatively weak current magnitude. The collective current direction is from the top to the bottom. Observing from these two figures, the direction of the current rotation is right-handed as the time increases, which stands for the RHCP radiation. Owing to the symmetry of the antenna and the feed ports, almost the same current distributions can also be observed when port 2 is excited, as shown in Fig. 4 (a) and (b). However, different from Fig. 4 (a), the direction of the current rotation for the last two figures is left-handed as the time increases, which means LHCP radiation is realized. From the observation of the current distributions, it can be seen that four crossed dipoles are 


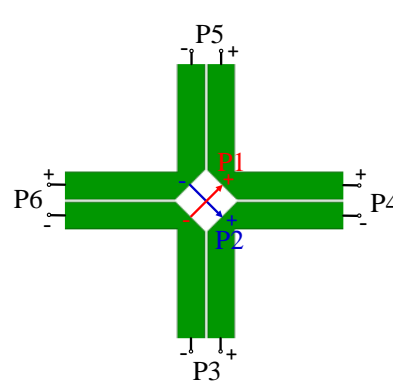

(a)

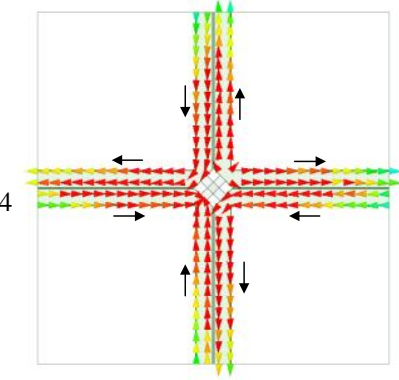

(b)

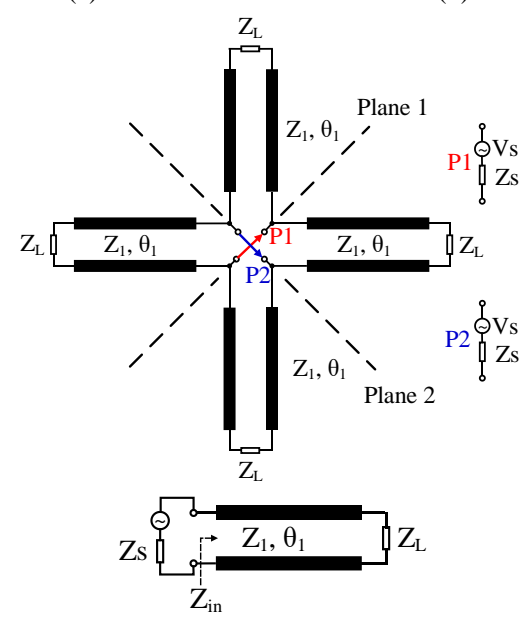

(c)

(d)

Fig. 5. (a) Simulation model of the OPD. (b) Current distribution on the surface of the OPD when port 1 is excited. (c) Equivalent circuit of the OPD. (d) Simplified equivalent circuit of the OPD when port 1 is excited.

sequentially excited at different times of the oscillation period, and DCP radiations are realized by exciting different ports.

To illustrate the working principle of the OPD, Fig. 5 (a) shows the simulation model of OPD extracted from the presented antenna. The OPD is composed of four pairs of coupled coplanar striplines. At the center of the model, two crossed ideal lumped ports (ports 1 and 2) are inserted to excite the OPD and defined as the input ports. Four wave ports (ports $3,4,5$, and 6) are located at the peripheral of the model and defined as the output ports. Fig. 5 (b) shows the current distribution on the surface of the OPD when port 1 is excited. As can be seen that the current directions on the coupled coplanar striplines are opposite. This means that transmission line mode is formed on these coupled coplanar striplines. Based on this, the equivalent circuit of the OPD is shown in Fig. 5 (c), which can be equivalent as four cascading transmission lines with the characteristic impedance of $Z_{1}$ and electric length of $\theta_{1}$. The OPD is terminated with the loads of $Z_{L}$. Two orthogonal input ports are located at the junction of the cascading points with the source impedance of $Z_{S}$. As can be seen that the equivalent circuit has two symmetrical planes (plane 1 and plane 2). Based on the equivalent circuit analysis, when port 1 is excited, the symmetrical plane 1 is equivalent as the open-circuited plane, while the symmetrical plane 2 is equivalent as the short-circuited plane. Therefore, when port 1 is excited, the corresponding simplified equivalent circuit is obtained and shown in Fig. 5 (d). As can be seen from the

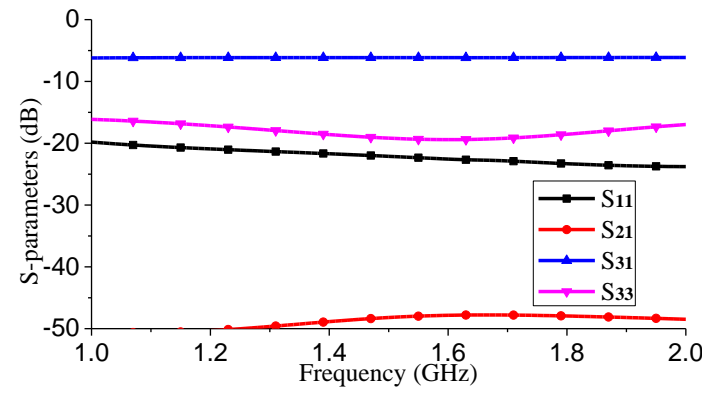

(a)

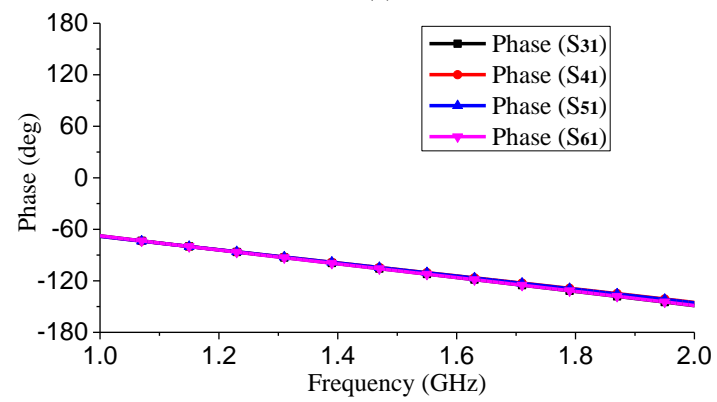

(b)

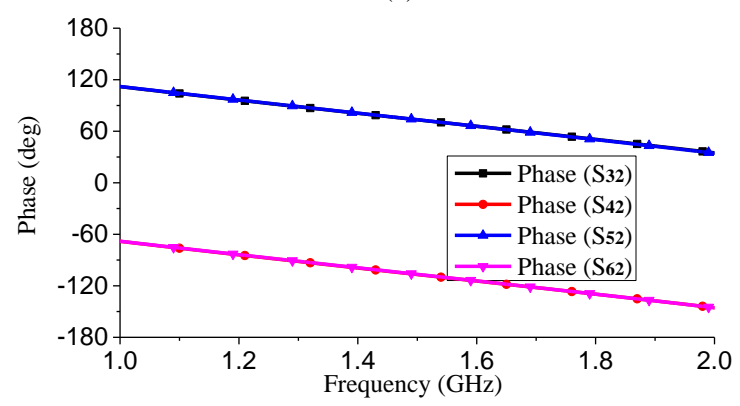

(c)

Fig. 6. Simulated results of the OPD. (a) Simulated S-parameters. (b) Transmission phase from port 1. (c) Transmission phase from port 2.

simplified equivalent circuit, the input impedance at the input port is

$$
Z_{\text {in }}=Z_{1} \frac{Z_{L}+j Z_{1} \tan \theta_{1}}{Z_{1}+j Z_{L} \tan \theta_{1}}
$$

and the corresponding reflection coefficient is

$$
\Gamma_{\text {in }}=\frac{Z_{\text {in }}-Z_{s}}{Z_{\text {in }}+Z_{s}}
$$

From (1)-(2), the input impedance and the reflection coefficient at the input port can be adjusted by $Z_{1}$ and $\theta_{1}$. It should be noted that, when $Z_{1}=Z_{L}=Z_{S}$, the input ports of the OPD will be naturally matched. In addition, if $Z_{L} \neq Z_{S}$, the transmission line with the characteristic impedance $Z_{1}$ and electric length $\theta_{1}$ can work as the impedance transformer when $\theta_{1}$ is designed as $90^{\circ}$. This can provide much flexibility to design the proposed wideband DCP antenna.

Fig. 6 shows the simulated results of the presented OPD. Owing to the symmetry of the OPD, the simulated magnitudes of $S_{11}$ and $S_{22}$ are almost the same, the simulated magnitudes of $\mathrm{S}_{31}, \mathrm{~S}_{41}, \mathrm{~S}_{51}, \mathrm{~S}_{61}, \mathrm{~S}_{32}, \mathrm{~S}_{42}, \mathrm{~S}_{52}$, and $\mathrm{S}_{62}$ are almost same, and the simulated magnitudes of $S_{33}, S_{44}, S_{55}$, and $S_{66}$ are almost same. Therefore, only $S_{11}, S_{31}$, and $S_{33}$ are given in Fig. 6 (a) for 


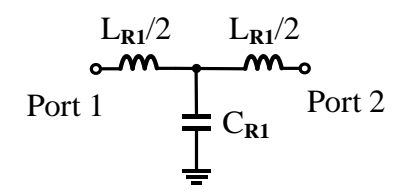

(a)
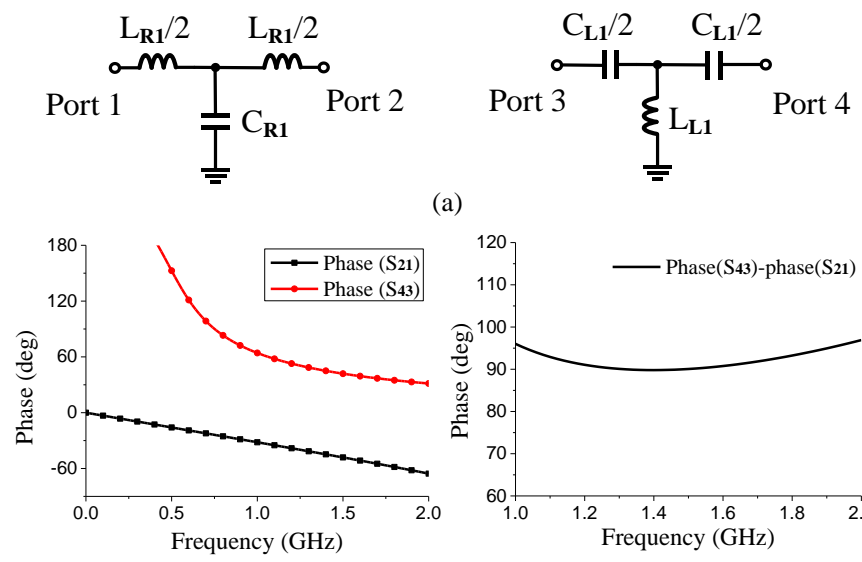

(b)

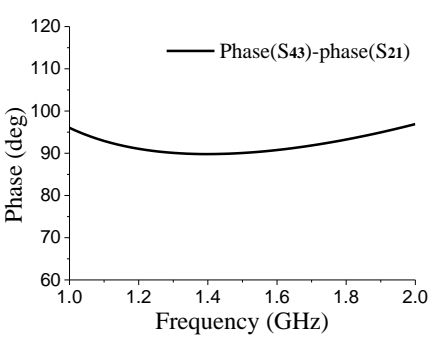

(c)

Fig. 7. (a) T-type RH-TL unit cell and T-type LH-TL unit cell. (b) Phase response of the two unit cells. (c) Phase difference between the two unit cells.

brevity. It can be seen that all the input and output ports are well matched, and the two input ports are isolated. Fig. 6 (b)-(c) show the different transmission phases from different input ports. It can be seen that, when port 1 is excited, all the outputs are in-phase. When port 2 is excited, signals from port 3 and port 5 are in-phase, but out-of-phase as compared to the port 4 and port 6. According to the simulated results in Fig. 6, the scattering matrix for the OPD can be concluded as

$$
[S]=\left[\begin{array}{cccccc}
0 & 0 & 0.5 & 0.5 & 0.5 & 0.5 \\
0 & 0 & -0.5 & 0.5 & -0.5 & 0.5 \\
0.5 & -0.5 & 0 & 0.5 & 0 & -0.5 \\
0.5 & 0.5 & 0.5 & 0 & -0.5 & 0 \\
0.5 & -0.5 & 0 & -0.5 & 0 & 0.5 \\
0.5 & 0.5 & -0.5 & 0 & 0.5 & 0
\end{array}\right]
$$

Observing from (3), because of the transmission phase characteristic at its output ports, it cannot be directly used to design DCP antenna. Therefore, $\pm 45^{\circ}$ phase shifters are needed to elaborately introduced and incorporated at the ends of the OPD to obtain the desired sequential quadrature phase excitations for DCP radiation. Four phase shifters are realized by using the T-type RH-TL unit cells and T-type LH-TL unit cells [24]. Fig. 7 (a) shows the T-type RH-TL unit cell and LH-TL unit cell. The corresponding phase shifts of these two unit cells are calculated as

$$
\begin{aligned}
& \phi_{R} \approx-2 \pi f \sqrt{L_{R 1} C_{R 1}} \\
& \phi_{L} \approx \sqrt{L_{L 1} C_{L 1}} /(2 \pi f)
\end{aligned}
$$

In this design, $\phi_{R}$ is designed for $-45^{\circ}$ phase shift and $\phi_{L}$ is designed for $+45^{\circ}$ phase shift at $1.4 \mathrm{GHz}$ to keep the antenna structure symmetrical for DCP radiation. According to (4)-(5), values of these lumped elements $L_{R 1}=2.6 \mathrm{nH}, C_{R 1}=1.5 \mathrm{pF}$, $L_{L 1}=10 \mathrm{nH}$, and $C_{L 1}=4.6 \mathrm{pF}$ are obtained. The calculated phase responses of the two unit cells are shown in Fig. 7 (b)-(c). As shown in the figure, the delayed phase for RH-TL unit cell is $-45^{\circ}$ at $1.4 \mathrm{GHz}$, and the delayed phase for LH-TL unit cell is $+45^{\circ}$ at $1.4 \mathrm{GHz}$. The phase difference between the two unit cells is $90-96^{\circ}$ from $1 \mathrm{GHz}$ to $2 \mathrm{GHz}$. Wideband quadrature phase and small phase variance are obtained. Therefore, wideband quadrature phase shift and small phase variance can

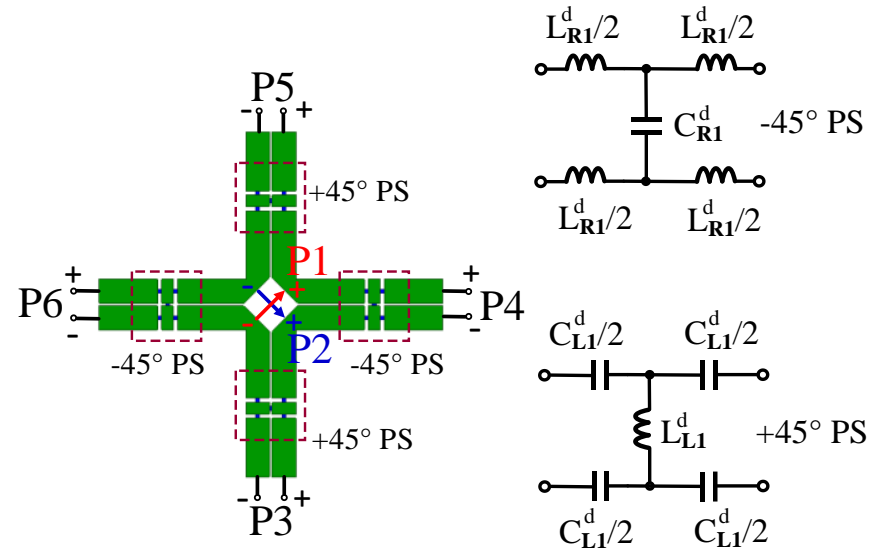

(a)

(b)

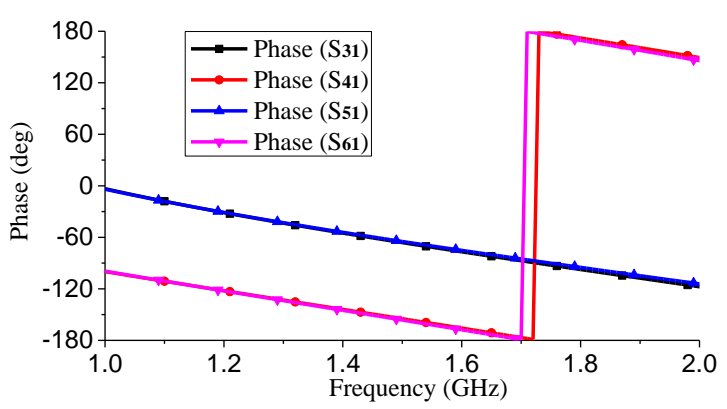

(c)

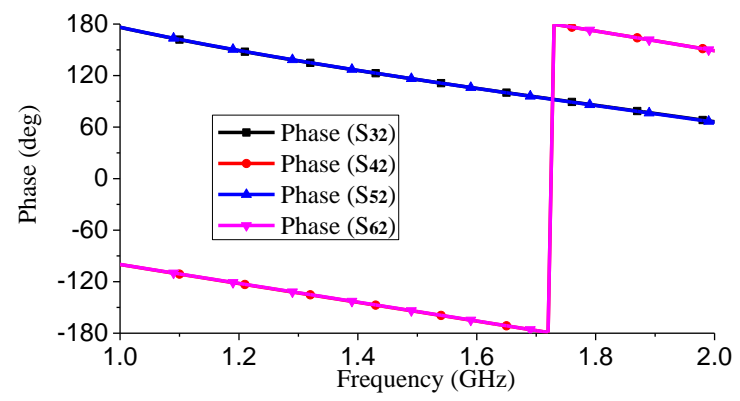

(d)

Fig. 8. The integrated feed network incorporating with OPD and $\pm 45^{\circ}$ phase shifters. (a) Simulation model. (b) Differential RH-TL unit cell and differential LH-TL unit cell (c) Phase responses when port 1 is excited. (d) Phase responses when port 2 is excited.

also be expected after incorporating these two types of unit cells into the integrated feed network.

Fig. 8 (a) shows the simulation model of the integrated feed network incorporating with the OPD, two RH-TL unit cells for $+45^{\circ}$ phase shift, and two LH-TL unit cells for $-45^{\circ}$ phase shift. It should be noted that the unit cells in Fig. 7 are single-ended circuit. For the proposed integrated feed network, differential RH-TL unit cells and differential LH-TL unit cell are used, which are shown in Fig. 8 (b). The component values for the differential unit cells are calculated as

$$
\begin{aligned}
& L_{R 1}^{d}=L_{R 1} \\
& C_{R 1}^{d}=C_{R 1} / 2 \\
& C_{L 1}^{d}=C_{L 1} \\
& L_{L 1}^{d}=2 L_{L 1}
\end{aligned}
$$

Fig. 8 (c)-(d) shows the simulated phase responses of the integrated feed network. Two sets of orthogonal quadrature 


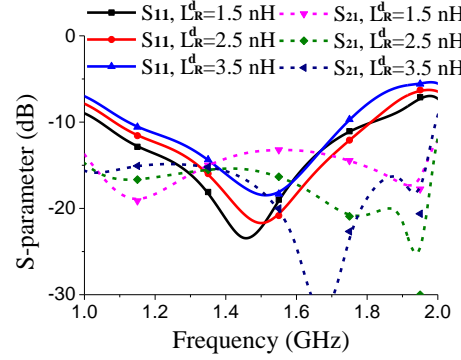

(a)

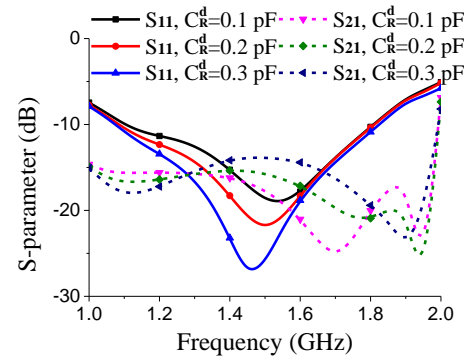

(c)

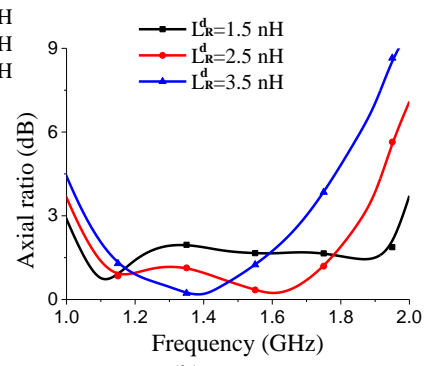

(b)

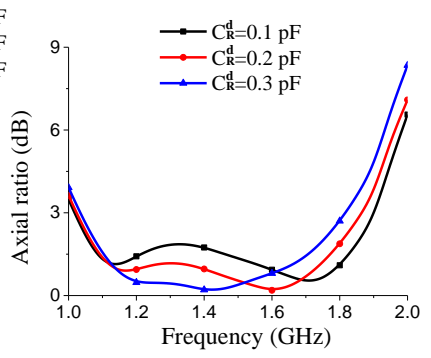

(d)

Fig. 9. The effect of the parameters of RH-TL unit cells on the performance of the proposed antenna. (a) S-parameters and (b) axial ratio vary with different $L_{R}^{d}$. (c) S-parameters and (d) axial ratio vary with different $C_{R}^{d}$.

phase responses are achieved when port 1 is excited and port 2 is excited, respectively. As shown in the figure, when port 1 is excited, signals at the port 4 and port 6 are in-phase, but lag $-90^{\circ}$ behind the port 3 and port 5 . Whereas when port 2 is excited, signals at the port 3 and port 5 are also in-phase, but lag $-90^{\circ}$ behind the port 4 and port 6 . Therefore, the scattering matrix for the integrated feed network combining with OPD and phase shifters in Fig. 8 can be concluded as

$$
[S]=\left[\begin{array}{cccccc}
0 & 0 & 0.5 j & 0.5 & 0.5 j & 0.5 \\
0 & 0 & -0.5 j & 0.5 & -0.5 j & 0.5 \\
0.5 j & -0.5 j & 0 & 0.5 & 0 & -0.5 \\
0.5 & 0.5 & 0.5 & 0 & -0.5 & 0 \\
0.5 j & -0.5 j & 0 & -0.5 & 0 & 0.5 \\
0.5 & 0.5 & -0.5 & 0 & 0.5 & 0
\end{array}\right]
$$

Compared to the matrix in (3), two sets of orthogonal quadrature signals are achieved at the output ports when the feed network is excited at the different input ports. Therefore, after incorporating four dipoles at the output ports, DCP radiation can be realized.

As discussed above, the component values of the RH-TL unit cell and LH-TL unit cell are important to realize DCP radiation. However, due to the distributed parasitic effects of the parallel transmission lines and the unideal input impedance of the four crossed dipoles, component values of discussed above provide the initial values to design the proposed DCP antenna. The optimal values of these lumped components are given in the caption of Fig. 2. Therefore, these parameters are studied to provide a guidance to design the proposed DCP antenna.

Fig. 9 shows the effect of the parameters of the RH-TL unit cells on the performance of the proposed antenna. Owing to the symmetry of the antenna, reflection coefficients at the two input ports are almost the same. So, only the reflection coefficient at port 1 is given in the figure. In Fig. 9 (a), as the increase of $L_{R}^{d}$, the center resonant frequency shifts to the upper

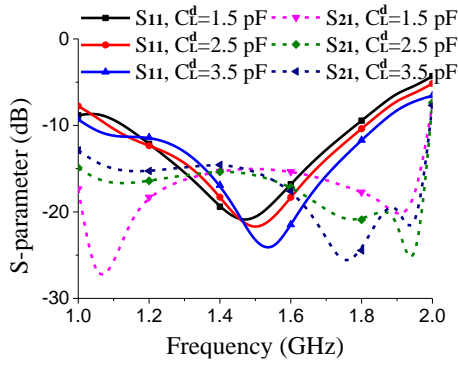

(a)

(c)

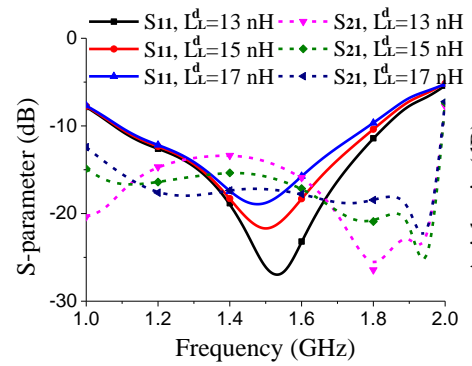

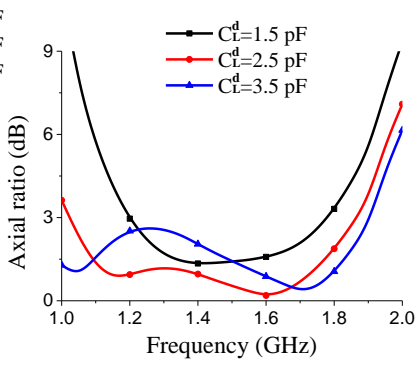

(b)

(d)

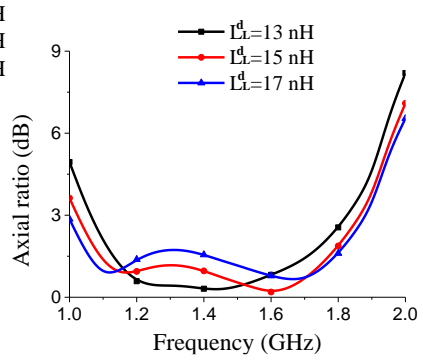

Fig. 10. The effect of the parameters of LH-TL unit cells on the performance of the proposed antenna. (a) S-parameters and (b) axial ratio vary with different $C_{L}^{d}$. (c) S-parameters and (d) axial ratio vary with different $L_{L}^{d}$.

band, which causes the impedance bandwidth becomes a little narrower. Compared to the variance of the reflection coefficient, the isolation between the two ports changes a lot, especially at the upper band. In Fig. 9 (b), as the increase of $L_{R}^{d}$, the axial ratio at the center frequency becomes lower. However, the bandwidth becomes narrower, and the axial ratio at the upper band changes obviously. In Fig. 9 (c), the impedance bandwidth for $S_{11}<-10 \mathrm{~dB}$ is almost unchanged as the increase of $C_{R}^{d}$, but the value of the reflection at the center becomes smaller. Isolation also changes a lot at the upper band as compared to the lower band. Similarly, the axial ratio in Fig. 9 (d) at the upper band changes obviously, and the bandwidth for axial ratio becomes narrower as the increase of $C_{R}^{d}$. Observing from the results from these four figures, the component values of the RH-TL unit cells mainly affect the isolation and the axial ratio at the upper band for the proposed antenna.

Fig. 10 shows the effect of the parameters of LH-TL unit cells on the performance of the proposed DCP antenna. As shown in Fig. 10 (a) and (b), when $C_{L}^{d}$ is increased from $1.5 \mathrm{pF}$ to $3.5 \mathrm{pF}$, the impedance bandwidth is slightly increased. However, the isolation at the lower band becomes poorer. The axial ratio at the lower band changes obviously as compared to the upper band. Similar effects can be found for $L_{L}^{d}$, as shown in Fig. 10 (c) and (d). The isolation at the lower band becomes poorer as the increase of $L_{L}^{d}$. The axial ratio at the lower band changes a lot compared to the upper band. Therefore, the component values of the LH-TL unit cells mainly affect the isolation and axial ratio at the lower band for the proposed antenna. Observing from the simulation results shown in Fig. 9 and Fig. 10, lumped components in the RH-TL unit cells and LH-TL unit cells have more serious effect on the isolation and AR performances as compared to the reflection coefficient for the presented antenna. By studying the effects of these parameters of the RH-TL unit cells and LH-TL unit cells on the 


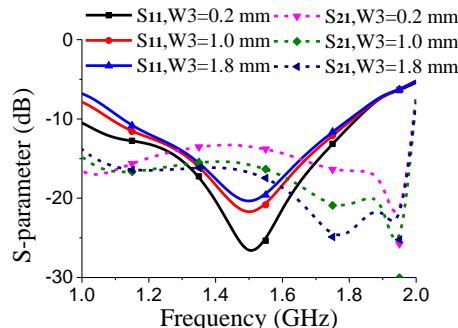

(a)

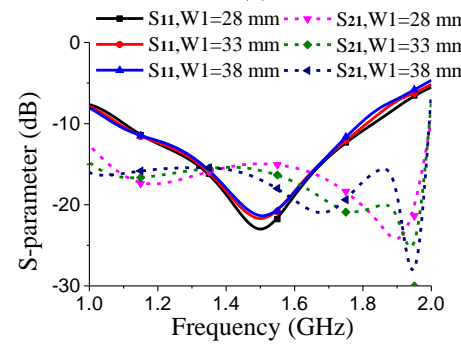

(a)

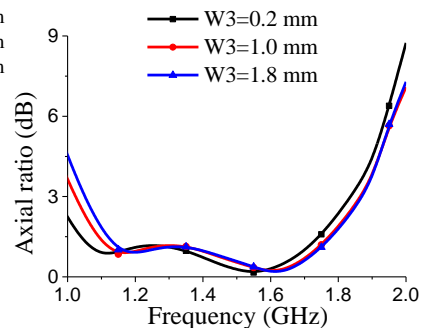

(b)

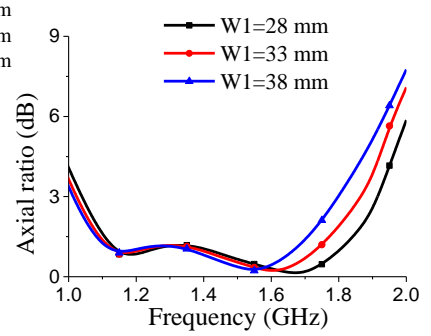

(b)

Fig. 11. The effects of the parameters of antenna configuration on the performance of the proposed antenna. (a) S-parameters and (b) axial ratio vary with different W3. (c) S-parameters and (d) axial ratio vary with different W1.

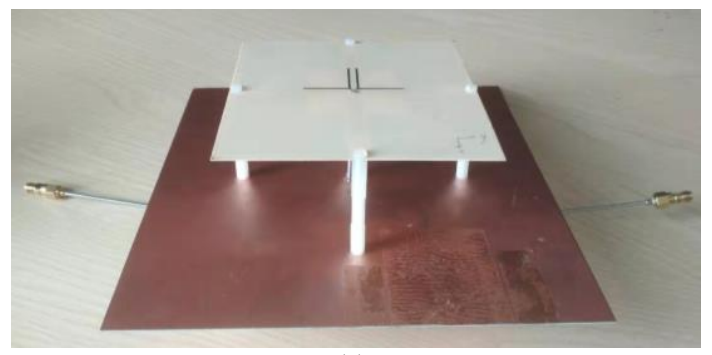

(a)

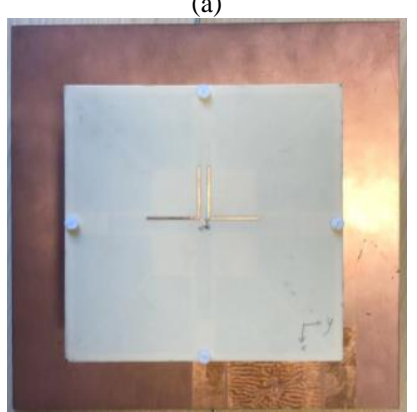

(b)

Fig. 12. Photographs of the fabricated prototype of the proposed DCP antenna. (a) $3 \mathrm{D}$ view. (b) Top view.

impedance and AR performances of antenna, it will not be difficult to design the proposed wideband DCP antenna.

Key parameters of the antenna geometry are also studied to provide guidance for the design of the presented DCP antenna. In the antenna configuration, slot width W3 and dipole width $\mathrm{W} 1$ determine the resonances of the crossed dipoles. Therefore, they are studied in Fig. 11 to show their effects on the antenna performances. As shown in Fig. 11 (a), when the slot width is decreased (that also means the dipole length is increased), the impedance bandwidth is increased, especially at the lower band. However, the isolation at the center band is deteriorated due to the increased bandwidth. The axial ratio in Fig. 11 (b) also shifts to the lower band as the decrease of the W3. Fig. 11 (c) and Fig. 11 (d) show the effects of dipole width W1 on the

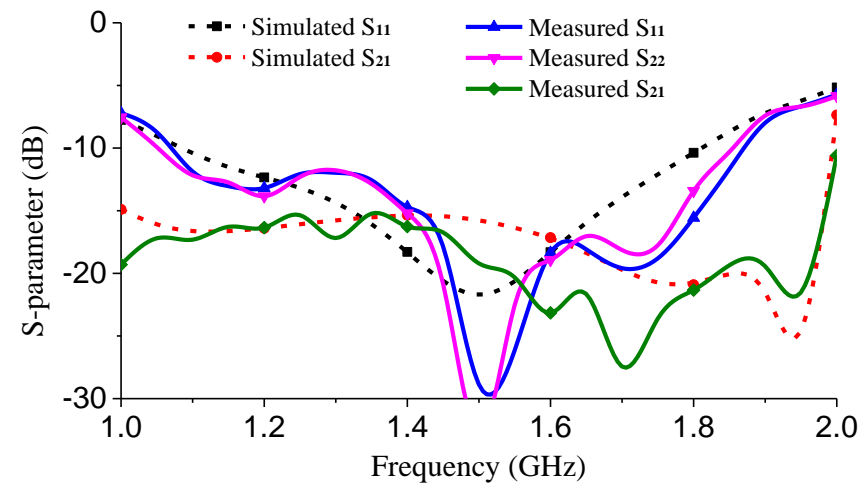

Fig. 13. Measured and simulated S-parameters of the proposed DCP antenna.

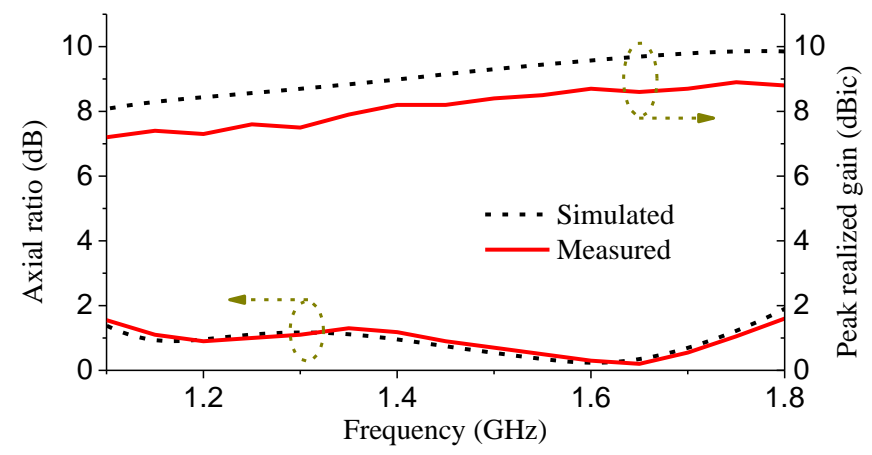

(a)

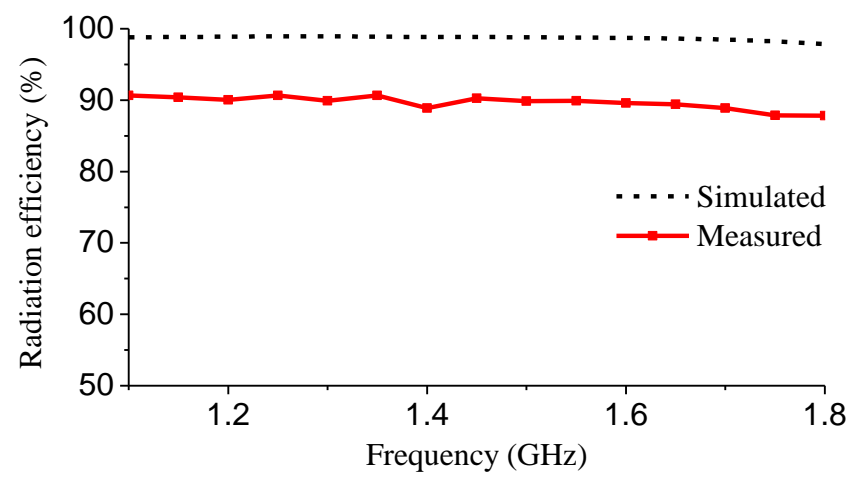

(b)

Fig. 14. Measured and simulated (a) axial ratio, peak realized gain, and (b) radiation efficiency of the fabricated prototype when RHCP radiation (port 1) is excited.

performances of the antenna. As the increase of W1, the impedance bandwidth is slightly increased, and shifts to the lower band. The isolation bandwidth of higher than $15 \mathrm{~dB}$ is increased accordingly. However, parameter W1 has significant effect on the axial ratio. The axial ratio bandwidth is decreased as the increase of $\mathrm{W} 1$, and the axial ratio is deteriorated a lot at the upper band. Therefore, to balance the bandwidth of both impedance and axial ratio, $\mathrm{W} 3=1 \mathrm{~mm}$ and $\mathrm{W} 1=33 \mathrm{~mm}$ are chosen for the DCP antenna design.

\section{RESULTS AND DisCUSSION}

\section{A. Antenna Verification}

The proposed DCP antenna was designed, fabricated, and measured to validate the above design method. The photographs of the fabricated prototype are shown in Fig. 12. The S-parameters of the antenna were measured by the Anritsu 

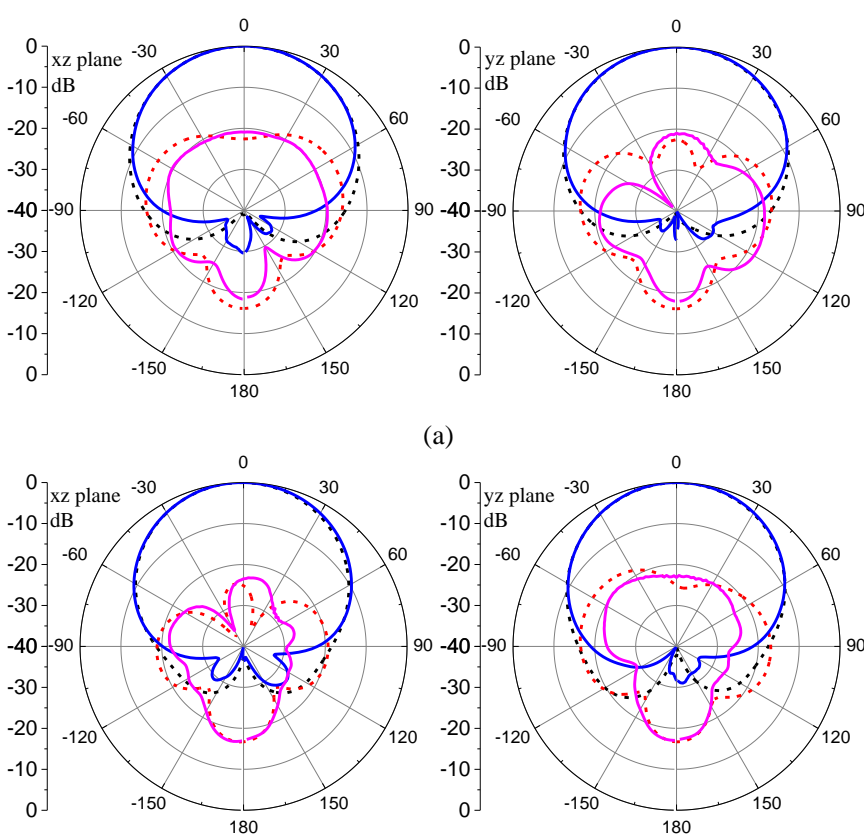

(a)

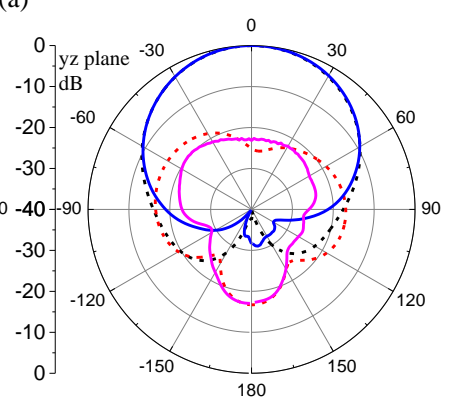

(b)
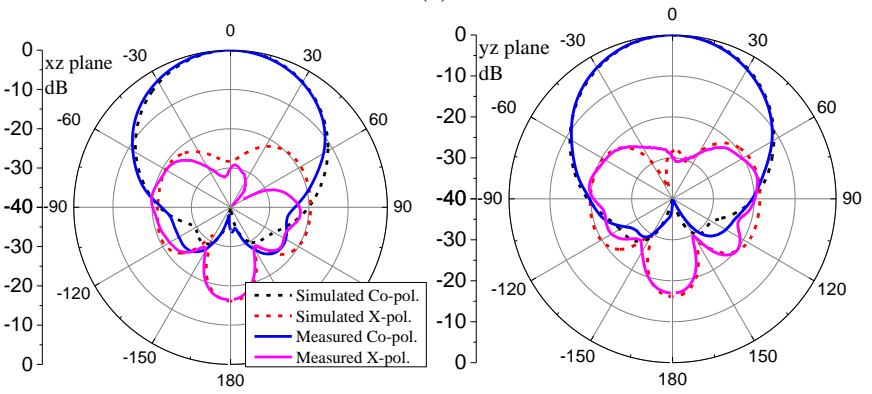

(c)

Fig. 15. Measured and simulated normalized radiation patterns at (a) $1.1 \mathrm{GHz}$, (b) $1.4 \mathrm{GHz}$, and (c) $1.7 \mathrm{GHz}$ when RHCP radiation (port 1) is excited. LHCP radiation is the cross-polarization.

37397C vector network analyzer, and the far field radiation patterns were measured by the ASYSOL far field measurement system at University of Kent. Fig. 13 shows the measured S-parameters of the presented antenna, which are compared with the simulated results. Good agreement can be observed between the simulated and measured S-parameters. The measured overlapped impedance bandwidth for both two input ports is from $1.07 \mathrm{GHz}$ to $1.85 \mathrm{GHz}$ with the reflection coefficients lower than $-10 \mathrm{~dB}$, which is slightly wider than the simulated impedance bandwidth. The corresponding isolation between the two input ports is higher than $15.2 \mathrm{~dB}$ within the bandwidth.

Fig. 14 shows the measured and simulated AR, peak realized gain, and radiation efficiency of the fabricated antenna prototype when RHCP radiation (port 1) is excited. Owing to the symmetry of the antenna, only RHCP axial ratio and gain are given in the figure. As shown in the figure, the measured AR is less than $1.7 \mathrm{~dB}$ from $1.1 \mathrm{GHz}$ to $1.8 \mathrm{GHz}$ within the bandwidth. Stable peak realized gain is achieved, which varies from 7.2-8.8 dBic. In addition, Stable radiation efficiency is achieved for the fabricated antenna. Due to the incorporated lumped element based LH-TL and RH-TL phase shift elements, the measured radiation efficiency is slightly lower than the
TABLE I

COMPARISON OF THE RECENTLY PUBLISHed DCP ANTENNAS

\begin{tabular}{|c|c|c|c|c|c|c|c|}
\hline Ref. & $\begin{array}{l}\text { Overlapped } \\
\text { BW (GHz) }\end{array}$ & $\begin{array}{l}\mathrm{AR} \\
(\mathrm{dB})\end{array}$ & $\begin{array}{c}S_{21} \\
(\mathrm{~dB})\end{array}$ & Radiation & Pattern & $\begin{array}{c}\text { Feed } \\
\text { network }\end{array}$ & Radiator \\
\hline [16] & $\begin{array}{l}4.9-5.95 \\
(19.4 \%)\end{array}$ & $<3$ & $<-16$ & UD & Sym. & Integrated & Patch \\
\hline [17] & $\begin{array}{l}9.4-11.0 \\
(15.7 \%)\end{array}$ & $<3$ & $<-15$ & UD & Sym. & Integrated & Patch \\
\hline [18] & $\begin{array}{c}3.5-4.0 \\
(16 \%)\end{array}$ & $<3$ & $<-10$ & UD & Sym. & Integrated & Patch \\
\hline [19] & $\begin{array}{c}0.8-0.97 \\
(19 \%)\end{array}$ & $<3$ & $<-15$ & UD & Sym. & Additional & Patch \\
\hline [20] & $\begin{array}{c}1.3-3 \\
(79 \%)\end{array}$ & $<3$ & I & UD & Sym. & Additional & 1 Dipole \\
\hline [21] & $\begin{array}{l}3.74-8.8 \\
(80.7 \%)\end{array}$ & $<3$ & $<-20$ & $\mathrm{BD}$ & Asym. & No & Monopole \\
\hline [22] & $\begin{array}{c}2-3.7 \\
(60 \%)\end{array}$ & $<3$ & $<-15$ & $\mathrm{BD}$ & Asym. & No & Slot \\
\hline [23] & $\begin{array}{l}1.83-6.35 \\
(110.5 \%)\end{array}$ & $<3$ & $<-10$ & $\mathrm{BD}$ & Asym. & No & Slot \\
\hline $\begin{array}{l}\text { This } \\
\text { work }\end{array}$ & $\begin{array}{c}1.07-1.85 \\
(53.4 \%)\end{array}$ & $<1.7$ & $<-15.2$ & UD & Sym. & Integrated & Dipole \\
\hline
\end{tabular}

simulated result. However, it is still very good and high than $87 \%$ within the bandwidth. The measured normalized radiation patterns at the frequency of $1.1 \mathrm{GHz}, 1.4 \mathrm{GHz}$, and $1.7 \mathrm{GHz}$ are shown in Fig. 15, which are compared with the corresponding simulated radiation patterns. As shown in these figures, symmetrical RHCP radiation is achieved when port 1 is excited, and LHCP radiation is the cross-polarization. Good accordance is observed between the simulated and measured radiation patterns. The measured half-power beamwidth is $60-70^{\circ}$ in xz-plane, and $60-72^{\circ}$ in yz-plane. The beamwidth becomes gradually narrower as the increase of the frequency. Therefore, higher antenna gain is achieved at the upper frequency. Because of the reflection of the antenna reflector, low back-lobe level is achieved with the measured front-to-back ratio higher than $16.4 \mathrm{~dB}$. The slight difference between the measured and simulated results, including the S-parameters and radiation patterns, may be caused by the tolerance of inductors and capacitors, fabrication errors, and position errors in the anechoic chamber.

\section{B. Comparison}

Table I compares the recently published DCP antennas with the presented antenna. DCP radiation can be realized by using patch antennas [16]-[20] with unidirectional and symmetrical radiation patterns. With the help of multi-coupling slots [16], series-fed method [17]-[18], or the additionally introduced branchline coupler [19], medium overlapped bandwidth for both impedance and AR is achieved. However, the bandwidth is still limited for the increasingly wider bandwidth required wireless communication systems. Monopole antennas [21] and slot antennas [22]-[23] provide ways to realize very wider bandwidth for DCP radiation. However, these antennas normally have bidirectional and asymmetrical radiation patterns. In addition, it is observed that the radiation patterns of these antennas are unstable, and the maximum radiation direction varies with the frequency. Obviously, it is difficult to use these antennas for array designs. Backed by reflectors, dipoles antenna in [20] has much wide bandwidth for DCP 
radiation with unidirectional and symmetrical radiation patterns. However, additional wideband quadrature coupler with four branchlines are utilized to provide required quadrature signals for DCP radiation. This will undoubtedly cause undesired insertion loss and increase the design cost. In this work, by using the integrated orthogonal feed network, the presented DCP antenna has the overlapped bandwidth of $53.4 \%$ for both impedance and AR requirements, which can provide enough operation bandwidth for ITS applications. Moreover, much lower AR $(<1.7 \mathrm{~dB})$ and unidirectional and symmetrical radiation patterns are achieved within the bandwidth.

\section{CONCLUSION}

This paper presents a novel method to design a wideband DCP antenna for ITS applications. Detailed equivalent circuit analysis illustrates that the integrated feed network can provide two sets of orthogonal equal magnitude and quadrature phase signals to the crossed dipoles for DCP radiation. The presented antenna is composed of an OPD with orthogonal input ports, four phase shifters for $\pm 45^{\circ}$ phase shift, and four crossed dipoles for DCP radiation. In the integrated feed network, the OPD is formed by the coupled coplanar striplines, and can provide equal magnitude in-phase and out-of-phase outputs. Whereas the $\pm 45^{\circ}$ phase shifters are designed by using the lumped element based differential LH-TL unit cell and RH-TL unit cell. A prototype of the proposed antenna was design, fabricated, and measured for verification. The measured results demonstrate that the presented antenna has a wide impedance bandwidth $(53.4 \%)$, low AR $(<1.7 \mathrm{~dB})$, and unidirectional symmetrical radiation patterns. Both the simulated and measured results show that the proposed DCP antenna can be a good front-end radiating component for ITS applications.

\section{REFERENCES}

[1] X. Lin, X. Sun, P. Ho, and X. Shen, "GSIS: A secure and privacy-preserving protocol for vehicular communications," IEEE Trans. Veh. Technol., vol. 56, no. 6, pp. 3442-3456, Nov. 2007.

[2] K. Mahler, W. Keusgen, F. Tufvesson, T. Zemen, and G. Caire, "Tracking of wideband multipath components in a vehicular communication scenario," IEEE Trans. Veh. Technol., vol. 66, no. 1, pp. 15-25, Jul. 2017.

[3] W.Viriyasitavat, M. Boban, H. M. Tsai, and A.Vasilakos, "Vehicular communications: survey and challenges of channel and propagation models," IEEE Veh. Technol. Mag., vol. 10, no. 2, pp. 55-66, Feb. 2015.

[4] S. Karagiannis et al., "Vehicular Networking: A survey and tutorial on requirements, architectures, challenges, standards and solutions," IEEE Commun. Surveys Tuts., vol. 13, no. 4, pp. 584-616, Apr. 2011.

[5] C. Cseh, "Architecture of the dedicated short-range communications (DSRC) protocol," Proc. 48th IEEE Veh. Technol. Conf., Ottawa, ON, Canada, 1998, vol. 3, pp. 2095-2099.

[6] M. Boban and P. M. d'Orey, "Exploring the practical limits of cooperative awareness in vehicular communications," IEEE Trans. Veh. Technol., vol. 65, no. 6, pp. 3904-3916, Dec. 2016.

[7] Z. Zhong et al., "A compact dual-band circularly polarized antenna with wide axial-ratio beamwidth for vehicle GPS satellite navigation application," IEEE Trans. Veh. Technol., vol. 68, no. 9, pp. 8683-8692, Sept. 2019.

[8] T. Varum, J. N. Matos, P. Pinho, and R. Abreu, "Nonuniform broadband circularly polarized antenna array for vehicular communications," IEEE Trans. Veh. Technol., vol. 65, no. 9, pp. 7219-7227, Sept. 2016.

[9] Q. Wu, Y. Zhou, and S. Guo, "An L-sleeve L-monopole antenna fitting a shark-fin module for vehicular LTE, WLAN, and Car-to-Car communications," IEEE Trans. Veh. Technol., vol. 67, no. 8, pp. 7170-7180, Aug. 2018.
[10] L. Ge, S. Gao, Y. Li, W. Qin, and J. Wang, "A low-profile dual-band antenna with different polarization and radiation properties over two bands for vehicular communications," IEEE Trans. Veh. Technol., vol. 68, no. 1, pp. 1004-1008, Jan. 2019.

[11] H. Wong, K. K. So, and X. Gao, "Bandwidth enhancement of a monopolar patch antenna with v-shaped slot for car-to-car and WLAN communications," IEEE Trans. Veh. Technol., vol. 65, no. 3, pp. 1130-1136, March 2016.

[12] C. Mao, S. Gao, and Y. Wang, "Dual-band full-duplex Tx/Rx antennas for vehicular communications," IEEE Trans. Veh. Technol., vol. 67, no. 5, pp. 4059-4070, May 2018.

[13] Q. Luo et al., "Multibeam dual-circularly polarized reflectarray for connected and autonomous vehicles," IEEE Trans. Veh. Technol., vol. 68, no. 4, pp. 3574-3585, April 2019.

[14] T. Mondal, S. Maity, R. Ghatak, and S. R. B. Chaudhuri, "Compact circularly polarized wide-beamwidth fern-fractal-shaped microstrip antenna for vehicular communication," IEEE Trans. Veh. Technol., vol. 67, no. 6, pp. 5126-5134, June 2018.

[15] L. Chi, Y. Qi, Z. Weng, W. Yu, and W. Zhuang, "A compact wideband slot-loop directional antenna for marine communication applications," IEEE Trans. Veh. Technol., vol. 68, no. 3, pp. 2401-2412, March 2019.

[16] C. Mao, S. S. Gao, Y. Wang, and J. T. Sri Sumantyo, "Compact Broadband Dual-Sense Circularly Polarized Microstrip Antenna/Array With Enhanced Isolation," IEEE Trans. Antennas Propag., vol. 65, no. 12, pp. 7073-7082, Dec. 2017.

[17] Y. Yang, J. Guo, B. Sun, Y. Cai, and G. Zhou, "The Design of Dual Circularly Polarized Series-Fed Arrays," IEEE Trans. Antennas Propag., vol. 67, no. 1, pp. 574-579, Jan. 2019.

[18] C. Zhang, X. Liang, X. Bai, J. Geng, and R. Jin, "A Broadband Dual Circularly Polarized Patch Antenna With Wide Beamwidth," IEEE Antennas Wireless Propag. Lett., vol. 13, pp. 1457-1460, 2014.

[19] X. Lai, Z. Xie, Q. Xie, and X. Cen, "A Dual Circularly Polarized RFID Reader Antenna With Wideband Isolation," IEEE Antennas Wireless Propag. Lett., vol. 12, pp. 1630-1633, 2013.

[20] H. Sun, H. Zhu, C. Ding, and Y. J. Guo, "Wideband Planarized Dual-Linearly-Polarized Dipole Antenna and Its Integration for Dual-Circularly-Polarized Radiation," IEEE Antennas Wireless Propag, Lett, vol. 17, no. 12, pp. 2289-2293, Dec. 2018.

[21] C. Ds and S. S. Karthikeyan, "A Novel Broadband Dual Circularly Polarized Microstrip-Fed Monopole Antenna," IEEE Trans. Antennas Propag., vol. 65, no. 3, pp. 1410-1415, March 2017.

[22] R. K. Saini and S. Dwari, "A Broadband Dual Circularly Polarized Square Slot Antenna," IEEE Trans. Antennas Propag., vol. 64, no. 1, pp. 290-294, Jan. 2016.

[23] R. Xu, J. Li, J. Yang, K. Wei, and Y. Qi, "A Design of U-Shaped Slot Antenna With Broadband Dual Circularly Polarized Radiation," IEEE Trans. Antennas Propag., vol. 65, no. 6, pp. 3217-3220, June 2017.

[24] I-Hsiang Lin, M. DeVincentis, C. Caloz, and T. Itoh, "Arbitrary dual-band components using composite right/left-handed transmission lines," IEEE Trans. Microw. Theory Techn., vol. 52, no. 4, pp. 1142-1149, April 2004.

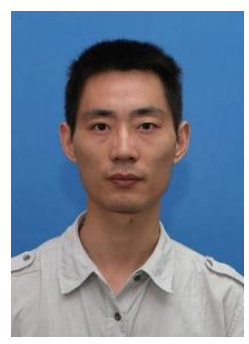

Lehu Wen received the B.S. in applied physics from Anhui University of Science and Technology, Huainan, China, in 2008, and the M.S. degree in electromagnetic fields and microwave technology from Xidian University, Xi'an, China, in 2011. He is currently working toward the Ph.D. degree with the University of Kent, Canterbury, U.K. His current research interests include base station antennas, mobile terminal antennas, and circularly polarized antennas. 


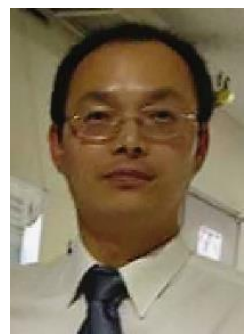

Steven Gao (M'01-SM'16-F'19) received the Ph.D. degree in microwave engineering from Shanghai University, Shanghai, China, in 1999.

$\mathrm{He}$ is a Full Professor and Chair in RF and Microwave Engineering, and the Director of Graduate Studies at the School of Engineering and Digital Arts, University of Kent, UK. His research covers smart antennas, phased arrays, MIMO, reconfigurable antennas, wideband multiband antennas, satellite antennas, RF microwave $\mathrm{mm}$-wave and $\mathrm{THz}$ circuits, mobile communications, satellite communications, UWB radars, synthetic-aperture radars, IOT and sensors for healthcare. He co-authored and co-edited three books (Space Antenna Handbook, Wiley, 2012 Circularly Polarized Antennas, IEEE-Wiley, 2014; Low-Cost Smart Antennas, Wiley, 2019), over 300 papers and 10 patents. He was a Distinguished Lecturer of IEEE AP Society, and is currently an Associate Editor of IEEE Transactions on Antennas and Propagation and several other international Journals (Radio Science, IEEE Access, Electronics Letters, IET Circuits, Devices and Systems, etc), and the Editor-in-Chief for John Wiley \& Sons Book Series on "Microwave and Wireless Technologies". He was General Chair of LAPC2013, and an Invited Speaker at many conferences. He is a Fellow of IEEE, and a Fellow of Royal Aeronautical Society, UK, and a Fellow of IET, UK.

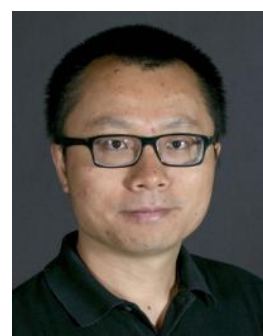

Qi Luo (S'08-M'12-SM'19) received the Ph.D. degree (with distinction) from the University of Porto, Porto, Portugal, in 2012. He is currently a Research Fellow with the School of Engineering and Digital Arts, University of Kent, Canterbury, U.K. His current research interests include smart antennas, circularly polarized antennas, reflectarray, transmitarray, multi-band microstrip antennas, and electrically small antennas. He has authored or coauthored two books, Circularly Polarized Antennas (Wiley-IEEE, 2014) and Low-Cost Smart Antennas (Wiley, 2019). Dr. Luo was awarded as the Outstanding Reviewer for the IEEE TRANSACTIONS ON ANTENNAS AND PROPAGATION in 2015. He has been serving as a reviewer for a number of technical journals and international conferences.

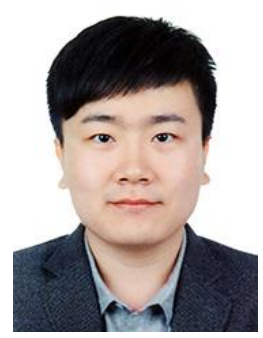

Wei Hu (S'09-M'13) received the Ph.D. degree in electromagnetic fields and microwave technology from Xidian University, Xi'an, China, in 2013. From 2013 to 2017, he was a Lecturer with the National Key Laboratory of Antennas and Microwave Technology, Collaborative Innovation Center of Information Sensing and Understanding, Xidian University, Xi'an, China, where he is currently an Associate Professor. From 2018 to 2019, he visited the University of Kent, U.K., as an Academic Visitor. He has authored and co-authored over 40 internationally refereed journal papers and has been serving as a reviewer for a number of technical journals and international conferences. His current research interests include multiband and wideband antennas, circularly polarized antennas, MIMO antenna arrays, and wideband wide-scanning phased arrays.

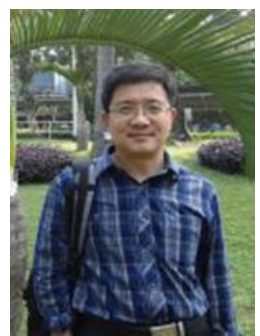

Yingzeng Yin (M'16) received the Ph.D. degree in electromagnetic wave and microwave technology from Xidian University, Xi'an, China, in 2002. From 1990 to 1992, he was a Research Assistant and an Instructor with the Institute of Antennas and Electromagnetic Scattering, Xidian University, where he was an Associate Professor with the Department of Electromagnetic Engineering from 1992 to 1996, and has been a Professor since 2004. His current research interests include the design of microstrip antennas, feeds for parabolic reflectors, artificial magnetic conductors, phased array antennas, and computer-aided design for antennas. 